\title{
Recoupement de la protection des droits de l'Homme et du droit international humanitaire (DIH) dans les situations de crise et de conflit
}

\author{
Hans-Joachim Heintze
}

\section{(2) OpenEdition Journals}

\section{Édition électronique}

URL : https://journals.openedition.org/conflits/1930

DOI : $10.4000 /$ conflits. 1930

ISSN : $1777-5345$

Éditeur :

CECLS - Centre d'études sur les conflits - Liberté et sécurité, L'Harmattan

\section{Édition imprimée}

Date de publication : 1 décembre 2005

Pagination : 123-147

ISBN : 2-296-00230-7

ISSN : 1157-996X

Référence électronique

Hans-Joachim Heintze, «Recoupement de la protection des droits de l'Homme et du droit international humanitaire (DIH) dans les situations de crise et de conflit », Cultures \& Conflits [En ligne], 60 | hiver 2005, mis en ligne le 23 février 2006, consulté le 27 septembre 2021. URL : http:// journals.openedition.org/conflits/1930; DOI : https://doi.org/10.4000/conflits.1930

Ce document a été généré automatiquement le 27 septembre 2021.

Creative Commons License 


\title{
Recoupement de la protection des droits de l'Homme et du droit international humanitaire (DIH) dans les situations de crise et de conflit
}

\author{
Hans-Joachim Heintze
}

1 Le droit international public classique opérait une nette distinction entre le droit international applicable en temps de paix et le droit international applicable en temps de guerre ${ }^{1}$. Suivant l'état des relations internationales on appliquait soit les normes du droit de paix, soit les normes du droit de guerre. Le droit de guerre s'appliquait dès la proclamation de l'état de guerre. A la fin de la guerre, le droit de paix trouvait à nouveau application.

2 Un des grands progrès civilisateur du droit international public moderne fut d'interdire aux Etats de faire usage de la force militaire afin d'imposer leurs intérêts nationaux. Cette interdiction avait déjà été prévue dans le Pacte Briand-Kellogg du 27 août 1928. L'article 1 dudit pacte dispose en effet que : «Les Hautes Parties contractantes déclarent solennellement au nom de leurs peuples respectifs qu'ils condamnent le recours à la guerre pour le règlement des différends internationaux et y renoncent en tant qu'instrument de politique nationale dans leurs relations mutuelles $»^{2}$. Cette disposition a été inscrite à l'article 2 (4) de la Charte des Nations-Unies de $1945^{3}$, et a désormais valeur de ius cogens.

3 La Charte des Nations-Unies prévoyait au demeurant de mettre à charge des Etats contractants, pour la première fois dans un traité international, l'obligation de respecter les droits de l'Homme. Ainsi, on a réussi à abandonner la très nette distinction entre droit de paix et droit de guerre, car la Charte prévoyait des normes applicables à la fois en temps de guerre et en temps de paix. Il s'agit des droits fondamentaux de l'Homme (comme, par exemple, le droit selon lequel nul ne peut être arbitrairement privé de sa vie) qui devaient être respectés en toutes circonstances. On 
étudiera par la suite la relation entre le droit international de guerre applicable en temps de conflit armé, plus communément appelé droit international humanitaire (DIH) et le droit des droits de l'Homme issu originairement du droit applicable en temps de paix.

L'application de principe du droit des droits de l'Homme en cas de guerre

Dans sa "Nuclear Weapons Advisory Opinion", la Cour Internationale de Justice (CIJ) rejette catégoriquement l'idée selon laquelle le Pacte international relatif aux droits civils et politiques (PIDCP) ${ }^{4}$, contenant les droits fondamentaux de l'Homme, ne s'appliquerait qu'en temps de paix ${ }^{5}$. La position de la CIJ trouve appui dans les termes des traités les plus importants relatifs aux droits de l'Homme et notamment à l'article 4 du PIDCP. Parmi ces dispositions, l'article 15 de la Convention européenne de sauvegarde des droits de l'Homme et des libertés fondamentales (CESDH) se réfère le plus explicitement à la situation juridique applicable lors de conflits armés. Selon cet article, il est possible de "déroger aux obligations prévues par la présente Convention » en cas de guerre ou en cas de danger public menaçant la vie de la nation. Il est possible de restreindre certains droits dans la stricte mesure où la situation l'exige. Cependant, certains droits, expressément énumérés (entre autres le droit à la vie, l'interdiction de torture, la liberté de croyance), ne peuvent faire l'objet d'aucune restriction, ce qui implique que les droits de l'Homme - auxquels on ne peut déroger - soient respectés en toutes circonstances. Par ce moyen, on franchit la traditionnelle frontière entre le droit de paix et le droit de guerre. Le droit des droits de l'Homme est devenu partie intégrante de la législation applicable en cas de conflit armé. Cerna en déduit très justement que le DIH « devient une branche du droit des droits de l'Homme, (...) des droits de $l^{\prime}$ Homme en situation de conflits armés $»^{6}$.

La codification du DIH à travers un traité des droits de l'Homme

5 La convention de 1989 sur les droits de l'enfant $(\mathrm{CDE})^{7}$ confirme cette vision de manière pertinente. Le chevauchement des champs d'application du droit des droits de l'Homme et du DIH devient alors évident. Selon l'article 38.1 de la CDE, les Etats partis s'engagent à respecter et à faire respecter les règles du DIH qui leur sont applicables en cas de conflit armé et dont la protection s'étend aux enfants. Une convention de droit de paix contient donc des règles de comportement en temps de guerre. Et ces règles sont encore davantage détaillées. Dans les alinéas suivants, on réitère les dispositions de l'article $77 \mathrm{du}$ protocole additionnel I aux Conventions de Genève (PA I) ${ }^{8}$ au sujet du recrutement et de la participation directe d'enfants aux conflits. Ces règles standard datant de 1977, autorisent le recrutement et la participation directe d'enfants à partir de l'âge de 15 ans.

6 Le fait que les règles standard du PA I, manifestement insatisfaisantes, soient reprises dans la CDE de 1989 va, d'un côté, à l'encontre d'une codification progressive du droit international et de l'autre, à l'encontre du but recherché de cette convention qui, selon l'article 3, doit être l'intérêt supérieur (« best interest ») de l'enfant ${ }^{9}$. Or, la participation directe d'un enfant de 15 ans à un conflit, en tant que soldat, ne sert certainement pas son intérêt supérieur. Dans la doctrine, cette contradiction a été maintes fois critiquée et a soulevé des questions relatives à la CDE de 1989. Pourquoi cette convention, qui fut pourtant élaborée douze ans après le PA I, ainsi qu'après un considérable mouvement de codification progressive des droits individuels, n'a-t-elle pas retenu une protection allant au-delà de celle garantie par l'article 77 du PA I ${ }^{10}$ ? Ce manque constaté est d'autant plus grave que les opposants à l'amélioration de ces droits de l'enfant (en 
particulier les Etats-Unis, l'Iran et l'Iraq) n'ont pas avancé d'argument juridique concluant au moment de la rédaction. Les Etats-Unis estimaient alors que ni l'Assemblée Générale ni la Commission des droits de l'Homme ne pouvaient être considérées comme légitimes pour réviser le DIH existant ${ }^{11}$.

7 L'argumentation américaine, bien qu'elle se situe dans la lignée de la traditionnelle séparation entre le droit de paix et le droit de guerre, ne convainc cependant guère. En effet, le CDE constituait à la base une nouvelle convention indépendante, dont l'objectif n'était nullement la révision du DIH mais plutôt l'obligation pour les Etats partis d'aller au-delà des règles générales, et, partant, d'offrir plus de garanties des droits, ce qui est, du point de vue du droit des contrats, parfaitement envisageable. Seul l'accord autour d'une protection moindre aurait été problématique. En ce sens, l'argumentation américaine tomberait donc à plat.

Mais les Etats-Unis ont finalement abandonné leur position indéfendable, comme en témoigne leur adhésion au protocole facultatif à la convention relative aux droits de l'enfant, concernant l'implication d'enfants dans les conflits armés. Ce protocole adopté en 2000 avec la résolution 54/263 de l'Assemblée Générale des Nations Unies oblige les Etats partis à prendre toutes les mesures possibles, afin d'empêcher la participation directe des enfants âgés de moins de 18 ans aux conflits. Il est entré en vigueur le 12 février 2002 et a été ratifié jusqu'à présent par cinquante-deux Etats. Désormais, au moins en ce qui concerne les Etats signataires, un standard supérieur à celui du DIH se trouve appliqué ${ }^{12}$.

9 L'exemple de la CDE montre non seulement le chevauchement entre le droit de paix et le droit de guerre, mais constitue également une preuve manifeste de l'impossibilité de contourner et de ne pas prendre en considération le droit international des droits de l'Homme, au moment de l'inventaire des différentes obligations incombant à un Etat en cas de conflit armé. Cet élément à lui tout seul justifie déjà le fait de parler d'une convergence des deux corps de droit.

L'application cumulative des droits de l'Homme et du DIH

10 Certaines obligations issues des conventions des droits de l'Homme restent en vigueur en situation de conflits armés. Cette circonstance ne trouve pas un écho unanime, que ce soit dans la pratique ou au sein de la doctrine. C'est avec une certaine retenue que le CICR et certains auteurs abordent le sujet en abondant dans le sens de la théorie de la complémentarité. Cette approche veut ainsi que les corps de droit des droits de l'Homme et du DIH soient non pas identiques, mais se complètent mutuellement et demeurent en fin de compte deux corps distincts ${ }^{13}$.

11 A côté de cette théorie de la complémentarité, une autre théorie plus offensive, la théorie de la convergence, gagne en importance. Cela se comprend aisément au vu des développements exposés ci-dessus concernant les conventions des droits de l'enfant ${ }^{14}$. La théorie de la convergence va au-delà de la simple complémentarité et vise la protection effective la plus large possible de la personne humaine par l'application cumulative des deux corps de droit, de telle sorte qu'on est amené à parler d'un corps unifié des droits de l'Homme au sein des différents chapeaux institutionnels ${ }^{15}$. Un tour d'horizon de la toute jeune pratique étatique dans ce domaine nous montre qu'il ne s'agit pas seulement d'un cas d'école.

12 Ainsi, Kälin nous fait remarquer que l'utilisation cumulative des deux corps de droit lors du conflit au Koweït fut tout aussi bien "réalisable " que «significatif », et démontre ainsi clairement la signification de la théorie de la convergence dans les 
conditions du régime d'occupation au Koweït de 1990-1991 ${ }^{16}$. Or, on peut aisément établir un parallèle avec la situation internationale contemporaine en Iraq. La Résolution 1483 (2003) relative à l'état d'occupation et à la reconstruction de l'Iraq d'aujourd'hui appelle ainsi tous les "participants » à s'acquitter pleinement de leurs obligations au regard du droit international, en particulier les obligations issues des conventions de Genève $\$ 5(\mathrm{CG})$, et oblige le représentant spécial du Secrétaire Général pour l'Iraq à assurer la promotion de la protection des droits de l'Homme (\$8 g). Un tel ensemble d'obligations relatif à un territoire d'occupation exige justement l'application cumulative du DIH et du droit des droits de l'Homme.

Cet aspect fait déjà l'objet d'enjeux et de disputes doctrinales, notamment à l'occasion d'autres situations d'occupation aux cours desquelles trois points ont été soulignés :

L'interprétation des droits et obligations nécessite le recours aux deux corps de droit. En effet, il est impossible d'interpréter l'expression droit-de-l'hommiste " traitement inhumain » dans un camp de prisonniers autrement qu'à la lumière des dispositions des CG III, car ce sont ces dernières qui lui confèrent une signification spécifique. D'un autre côté, on ne saurait satisfaire à l'exigence inscrite à l'article $3 \$ 1$ (d) commun aux quatre CG qui demande le respect, lors d'une procédure pénale « des garanties judiciaires reconnues comme indispensables par les peuples civilisés ", sans utiliser les instruments de protection des droits de l'Homme.

Les droits de l'Homme renforcent les règles du DIH en reformulant de manière plus exacte les obligations à charge des Etats partis. Il faut ainsi interpréter les engagements contenus à l'article $55 \mathrm{f}$ de la Convention de Genève (IV) concernant la santé à la lumière du droit à la santé inscrit dans le Pacte onusien relatif aux droits économiques, sociaux et culturels ${ }^{17}$. En ce qui concerne la distinction entre le viol (utilisé en tant que moyen de guerre et prohibé par le DIH) et la torture, il faut obligatoirement se référer aux dispositions relatives aux droits de l'Homme contenues dans l'accord ${ }^{18}$ contre toute forme de torture ${ }^{19}$.

16 Le DIH rend effectif le droit des droits de l'Homme. A titre d'exemple, il concrétise les obligations relatives aux personnes portées disparues. Bien que le fait de "faire disparaître " quelqu'un constitue, sans aucun doute, une grave violation des droits de l'Homme, le droit applicable aux obligations à charge des Etats dans un tel cas n'est que très peu développé. Les CG III et IV, en revanche, obligent les forces d'occupation à délivrer les informations au sujet de personnes détenues, et des possibles causes de décès, à libérer les personnes emprisonnées et encore en vie, et à effectuer des recherches sur le sort des autres ${ }^{20}$.

Dans son rapport au Conseil de Sécurité, On the Protect of Civilians in Armed Conflict, le Secrétaire Général des Nations Unies va également dans le sens d'une application cumulative de toutes les normes de protection individuelle, au moins en ce qui concerne les civils. De même, il conseille la ratification des principaux instruments du DIH, des droits de l'Homme et du droit des réfugiés, car ceux-ci constituent les « outils essentiels en vue de la protection des civils dans les conflits armés " ${ }^{21}$.

D'un point de vue pratique, enfin, la convergence vers le droit international des droits de l'Homme est certainement une conséquence de la multiplication des conflits interétatiques qui, en raison du faible encadrement normatif du DIH, se situaient dans une zone d'ombre de ce dernier ${ }^{22}$. Fleck a pointé de manière très convaincante l'importance pratique de cette question pour les parties au conflit ${ }^{23}$. 
Le DIH comme lex specialis

19 L'application cumulative des droits de l'Homme et du DIH soulève obligatoirement la question de leurs relations réciproques. Question à laquelle la $\mathrm{CIJ}$ a dû répondre dans la Nuclear Advisory Opinion ${ }^{24}$, suite à l'argumentation des défenseurs de l'utilisation illégale d'armes atomiques, qui estimaient que l'utilisation de telles armes violerait le droit à la vie tel qu'il est inscrit dans l'article 6 PIDCP $^{25}$. L'article 6 dispose que nul ne peut être arbitrairement privé de sa vie. Dans son rapport d'expertise, la CIJ constate que l'article 6 PIDCP constitue un droit auquel on ne peut déroger, et qui, par conséquent, s'applique également dans les conflits armés. L'interdiction de priver quelqu'un « intentionnellement» de sa vie trouve donc à s'appliquer aussi en cas de guerre. Néanmoins, la CIJ reconnaît la primauté du DIH en temps de guerre et qualifie ce dernier de lex specialis. Le terme «intentionnellement " doit donc être défini selon le droit international humanitaire. Le recours aux droits de l'Homme s'avère sans grande utilité dans ce cas. Prenons l'exemple suivant : un combattant qui, dans le cadre d'actes licites de guerre, tue un combattant ennemi, n'aura pas à répondre pénalement de cet acte en application du ius bello ${ }^{26}$. L'article 15.2 de la CESDH pointe explicitement ces mesures spécifiques de la protection des droits applicables en temps de guerre. Selon cet article, les décès résultant d'actes licites de guerre ne peuvent être considérés comme une violation au droit à la vie au sens de l'article $3 \mathrm{CESDH}$.

La doctrine a salué, à l'occasion de l'évaluation du rapport d'expertise de la CIJ, la mise au point concernant les normes élaborées pour être applicables en temps de paix, qui ne sauraient trouver application dans le cadre d'actes perpétrés en temps de guerre. Les droits de l'Homme devraient plutôt être intégrés de manière délicate à la structure $\mathrm{du} \mathrm{DIH}^{27}$. On rappellerait ainsi la primauté du DIH en situation de conflit. Il faut cependant souligner que les droits de l'Homme dans leur ensemble, correspondant aux objectifs de l'article 4 PIDCP (respectivement des traités régionaux correspondants), demeurent applicables et conservent par conséquent leur importance. C'est la raison pour laquelle la $\mathrm{CIJ}$ rappelle, dans son rapport d'expertise, la nécessité de considérer le DIH ainsi que la protection des droits de l'Homme en tant qu'unité, et la nécessité de procéder à leur harmonisation.

21 En adoptant un tel point de vue, on ne peut ignorer l'objection de la lex specialis derogat legis genera. Cette objection peut cependant être contrée par le DIH lui-même : la clause Marten, adoptée sur la base du droit international coutumier comme de la pratique internationale conventionnelle stipule que les dispositions du droit international de guerre ne peuvent être perçues comme des règles à caractère absolu par rapport à la protection des droits de l'Homme. Elles sont, au contraire, complétées par les normes de protection des droits de l'Homme ${ }^{28}$. L'article 27 PA I témoigne également de "l'ouverture du droit international de guerre ${ }^{29}$. En effet, en ce qui concerne le traitement de personnes se trouvant sous l'emprise d'une des parties au conflit, l'article dispose que «les dispositions de la présente section complètent (...) les autres normes applicables au droit international qui régissent la protection des droits fondamentaux de l'homme pendant un conflit armé à caractère international ».

Les mécanismes de mise en œuvre de la protection des droits de l'Homme

22 La doctrine montre que la protection des droits de l'Homme partage non seulement une philosophie commune au DIH, mais qu'on peut également faire appel à elle pour compenser les déficits du $\mathrm{DIH}^{30}$. L'une des grandes faiblesses du DIH réside 
certainement dans le niveau peu développé de ses mécanismes de mise en œuvre, très peu efficaces.

C'est la raison pour laquelle le CICR et les auteurs ont toujours essayé de se servir des mécanismes de mise en œuvre des traités onusiens des droits de l'Homme, tels les conventions de contrôle de l'armement et les conventions sur l'environnement, en les utilisant en tant que modèle pour des procédures analogues concernant le DIH, dans le but de les rendre plus attractifs pour les Etats. Le système des procédures de rapports étatiques y occupe une place centrale ${ }^{31}$. Toutefois, la proposition de création de nouvelles procédures en matière de rapport ne semble présenter que très peu de chances d'aboutissement. Il faudrait plutôt concentrer toute l'attention autour de procédures déjà existantes et en faire des usages multiples. Des usages multiples paraissent possibles, en effet, grâce au chevauchement de la protection des droits de l'Homme et du DIH ${ }^{32}$.

L'obligation de rapportage en cas d'état d'urgence

24 L'expérience nous a montré que c'est surtout en temps de guerre ou suite à un autre danger public menaçant la vie de la nation, qu'on aboutit fréquemment à de graves violations des droits de l'Homme pouvant aller jusqu'à des exécutions de masses ${ }^{33}$. C'est pourquoi il est particulièrement important de contrôler le respect des droits de l'Homme dans ce genre de situation.

25 Ce défi répond aux exigences du PIDCP, à savoir l'obligation, selon l'article 4.2 pour les Etats, lorsque ceux-ci ont proclamé un état d'urgence, de signaler au Secrétaire Général de l'ONU les dispositions relatives aux droits de l'Homme auxquelles ils ont dérogé, ainsi que les motifs qui ont conduit à une telle dérogation. Le Comité des droits de l'Homme peut alors examiner les éléments constitutifs d'un état de danger public exceptionnel et, le cas échéant solliciter l'élaboration de rapports spéciaux ${ }^{34}$. En 1981 déjà, le Comité avait adopté une « déclaration générale » au sujet de l'interprétation des obligations inscrites à l'article $4^{35}$. Le Comité y soulignait la nature extraordinaire et temporaire du droit applicable à l'état d'urgence et engageait les Etats à rendre compte, sans délais, de chaque proclamation d'état d'urgence et à garantir le maintien de certains droits indérogeables. Le Comité intimait alors de ne prendre des mesures dérogeant aux droits de l'Homme que dans la stricte mesure où la situation l'exige. Le PIDCP visait par là le principe de proportionnalité, l'un des principes les plus importants du DIH.

26 De son côté, la CESDH ne connaît pas d'obligation de rapportage mais seulement une obligation d'information. Selon l'article 15.3, le Secrétaire Général du Conseil européen doit être informé des mesures prises lors de l'état de danger exceptionnel. Cette obligation n'est pas à traiter à la légère en raison de la possibilité de dépôt d'une plainte étatique, les déclarations étant publiées par le Conseil européen. L'article 27.3 de la déclaration américaine des droits de l'Homme est calqué sur ce modèle. Par conséquent celle-ci ne contient également qu'une obligation d'information vis-à-vis du Secrétaire Général de l'OAS (Organization of American States).

Le mécanisme de l'obligation de rapportage permet ainsi de surveiller l'observation des obligations relevant à la fois des droits de l'Homme et du DIH (dans la mesure où les deux corps de droit se recoupent), comme en témoigne le plus récent des rapports du Comité des droits de l'Homme à l'Assemblée Générale des Nations Unies : "face à des situations de conflits armés, internes comme externes, qui affecteraient les parties contractantes, le Comité examinera nécessairement si les parties remplissent ou non l'ensemble de leurs 
obligations issues de la Convention ${ }^{36}$. L'avantage de cette procédure réside dans le fait que les Etats partis doivent se justifier par rapport aux éventuelles dérogations aux droits de l'Homme. Toutefois, la multiplication de rapports périodiques à produire ne permet guère au Comité des droits de l'Homme de se consacrer entièrement à la notification des mesures prises sous le coup de l'Etat d'urgence. Cela ne va pas sans constituer un inconvénient non négligeable. Faut-il pour autant élaborer de nouveaux instruments de procédure? Une réorganisation des méthodes de travail permettrait très certainement de rendre la surveillance plus effective.

Plainte individuelle

Les victimes de violations du DIH ne disposent pas, au niveau universel, d'une procédure qui prend la forme d'une plainte individuelle. Toutefois, les procédures existantes en droit des droits de l'Homme permettent de compenser partiellement ce déficit. C'est ainsi que 104 des 149 Etats, qui ont non seulement ratifié le PIDCP mais également les protocoles facultatifs correspondants ${ }^{37}$, permettent aux victimes de violations des droits de l'Homme, après que celles-ci aient épuisées toutes les voies de recours interétatiques, de déposer une communication au Comité des droits de l'Homme. On vérifie lors de cette procédure si le/la plaignant(e) individuel(le) a effectivement subi une violation des droits de l'Homme. L'examen se restreint à vérifier si, selon l'article 1 du Protocole facultatif, il s'agit "d'une violation d'un des droits inscrits au Pacte ", ce qui signifie que le DIH n'est pas directement applicable. Cette procédure trouve toute son utilité en cas de danger public exceptionnel où les violations graves des droits de l'Homme peuvent être très fréquentes ${ }^{38}$. C'est la raison pour laquelle le contrôle international est de première importance. S'y ajoute le fait qu'on vérifie également, lors de cette procédure relative aux droits de l'Homme, si la limitation des droits du plaignant est en accord avec les exigences de l'article $4^{39}$. Cependant, la procédure n'aboutit pas à un jugement ${ }^{40}$ mais seulement à une prise de position du Comité. Néanmoins, grâce à la publication de la procédure on parvient à exercer une certaine pression sur l'Etat concerné (l'effet public blame).

Cette pression s'avère être plus forte selon les procédures de traités régionaux des droits de l'Homme, car ce sont alors des procédures judiciaires s'appliquent. Il faut souligner ici qu'on a déjà abouti par le passé, lors de telles procédures de plaintes individuelles, à l'application cumulative directe du DIH. C'est le résultat de l'article 15 al 1 de la CESDH qui stipule que les mesures prises en cas d'état d'urgence ne doivent pas être "en contradiction avec les autres obligations découlant du droit international ». L'article 27 de la convention américaine des droits de l'Homme connaît une formulation similaire. Un regard dans la pratique nous montre les avantages et les inconvénients de cette procédure.

30 La convention américaine des droits de l'Homme. Le fait même de savoir si les organes américains de protection des droits de l'Homme pouvaient faire appel au droit humanitaire pour rendre leurs décisions était source de conflit. En 1987, devant l'InterAmerican Commission on Human Rights et à l'occasion de l'affaire "Disabled Peoples' International et al. V. United States " relative à l'intervention de Grenade lors de laquelle seize patients d'une clinique psychiatrique furent entre autres blessés, le gouvernement a décidé que la Commission n'était pas l'organe compétent. L'argument déployé était le suivant: la Commission ne serait pas compétente pour juger de l'application de la CG IV, car son mandat se réduirait à l' " examen de la jouissance ou de la déprivation des droits inscrits dans l'American Declaration of Rights and Duties of 
Man $»^{41}$. Cette position défendue par les Etats-Unis fut critiquée en son temps par la doctrine selon laquelle les principes du DIH s'appliqueraient tout aussi bien à une opération militaire ${ }^{42}$.

31 La pratique ultérieure le confirme. Il convient de se référer en particulier au rapport d'expertise de l'Inter-American Commission on Human Rights dans sa décision "Tablada ", relative à l'attaque, par quarante-deux personnes armées, de la caserne des forces armées argentines La Tablada, le 30 octobre 1997. Vingt-neuf des attaquants et quelques soldats ont été tué lors des conflits qui ont duré trente heures. Les attaquants survivants s'adressent à la Commission et dénoncent les violations commises par l'Argentine, en vertu de la Convention américaine des droits de l'Homme et du DIH. Suite à cette plainte, la Commission a vérifié si elle pouvait appliquer directement le DIH et a fini par le faire. La doctrine a accordé une grande importance à cette décision, car cette dernière a confirmé le fait qu'un organe de protection des droits de l'Homme pouvait procéder à l'application directe du DIH vis-à-vis d'un Etat signataire d'une convention de protection des droits de l'Homme ${ }^{43}$. La Commission a justifié le recours au DIH par le fait que c'est le seul moyen d'action possible dont elle dispose face à des situations de conflits armés. Bien que d'un point de vue formel la Convention américaine des droits de l'Homme soit applicable lors de conflits armés, elle ne contient aucune réglementation relative aux moyens et méthodes de faire la guerre. Cependant, la Commission est obligée de se référer au DIH, afin de déterminer si il y a eu privation arbitraire de vie dans les conditions de guerre ${ }^{44}$.

32 Cette argumentation de la Commission est pertinente en droit. En effet, les seules dispositions de la Convention américaine des droits de l'Homme ne permettent pas de déterminer les personnes ayant pu participer légitimement aux hostilités et procéder à des actes de guerre. D'un autre côté, la Commission ne contient aucune disposition exigeant le report au DIH. La Commission justifie ainsi sa démarche par le recoupement des champs d'application du droit des droits de l'Homme et du DIH. Les Etats sont, de toute manière, aux termes de la convention des droits de l'Homme, soumis à l'obligation de respecter les dispositions de l'article 3 commun aux quatre CG :

33 - l'article $29 \mathrm{~b}$, non susceptible d'interprétation, qui stipule qu'on pourrait réduire le bénéfice ou l'exercice de certains droits qui sont garantis dans un autre accord liant l'Etat ;

34 - l'article 25, selon lequel chacun dispose du droit à un recours approprié en cas de violation de ses droits fondamentaux ;

35 - l'article 27, selon lequel les dérogations aux obligations inscrites à la convention ne peuvent pas être incompatibles avec les autres engagements internationaux souscrits ;

36 - le rapport d'expertise de la Cour interaméricaine des droits de l'Homme, selon lequel la Commission est autorisée à se référer également à d'autres conventions qui n'ont pas été élaborées au sein du système interaméricain ${ }^{45}$.

37 Les arguments sont de taille. La Commission a ainsi directement appliqué le DIH et ne s'est pas contentée de faire appel à lui comme outil d'interprétation.

38 La Cour interaméricaine des droits de l'Homme, dans sa décision "Los Palmeras " ${ }^{46}$, n’a pas suivi l'opinion de la Commission, à savoir qu'elle serait compétente pour appliquer directement le DIH et, en particulier, l'article 3 commun aux CG. La Cour a seulement admis qu'elle ne pourrait se référer aux CG que lorsqu'il s'agit de l'interprétation de la convention des droits de l'Homme. Dans sa décision «Los Palmeras», laquelle 
concernait l'exécution de six civils non armés par les policiers colombiens, la Cour a ainsi déclaré que la convention « a seulement donné compétence à la Cour pour déterminer si oui ou non les actes et les normes des Etats sont compatibles avec la Convention elle-même et non avec la Convention de Genève de $1949 »^{47}$.

Selon Kleffner et Zegveld, cette décision illustre la problématique qu'engendre l'affirmation de la Commission, selon laquelle elle pourrait appliquer directement le DIH. Etant donné les sérieux doutes quant à cette application directe, les auteurs suggèrent la nécessité de créer un mécanisme de procédure de plainte, propre aux violations du $\mathrm{DIH}^{48}$. Aussi souhaitable qu'une telle procédure puisse paraître, ses chances d'aboutir dans un futur proche sont extrêmement minces. La doctrine doit donc continuer à veiller à ce que les organes de protection des droits de l'Homme poursuivent la direction engagée par la décision "Tablada", afin de conférer davantage de poids au DIH ${ }^{49}$.

La Cour Interaméricaine $\mathrm{a}$, en revanche, abouti à un tout autre résultat dans sa décision "Bamaca-Velasquez $»^{50}$, ce qui relativise la critique de Kleffner et Zegveld concernant la décision «Los Palmeras ». La décision «Bamaca-Velasquez » est relative à la torture et à l'exécution d'un guérillero par des militaires guatémaltèques. Dans cette affaire, le DIH a été appliqué car le Guatemala, et la Commission était d'avis que le DIH trouverait application et que l'article $3 \mathrm{CG}$ pourrait servir à l'interprétation des obligations issues de la Convention américaine. La Cour a justifié sa démarche par le renvoi à l'article 29 de la Convention. Cet article autoriserait, au terme de l'interprétation, le recours à d'autres conventions que le Guatemala a signé, afin d'éviter une restriction inacceptable des droits de l'Homme. De manière explicite, elle a rajouté que l'existence établie d'un conflit armé interne signifie qu'" au lieu d'exonérer l'Etat de ses obligations à respecter et garantir les droits de l'Homme, ce fait le contraint à agir en accord avec de telles obligations $»^{51}$. Ce jugement confirme l'application directe du DIH par les cours chargées de la protection des droits de l'Homme et a mené la doctrine à la conclusion suivante : le DIH confirme la nécessité de punir les responsables de tels crimes ${ }^{52}$.

Convention européenne de sauvegarde des droits de l'Homme. L'article 15 de la CESDH n'autorise les dérogations relatives à des obligations conventionnelles que lorsque celles-ci sont en conformité avec les obligations internationales. Dans ce cadre, les obligations issues des CG sont donc à respecter ${ }^{53}$. Dans son arrêt «Irlande du Nord» ${ }^{54}$, la CEDH a en effet vérifié si les dérogations étaient en accord avec les autres obligations internationales contractées par l'Etat concerné. Plus précisément, la CEDH a vérifié si les lois britanniques applicables en Irlande du Nord étaient conformes aux CG. Cependant, ce contrôle ne fut pas approfondi pour cause d'éléments insuffisants fournis du côté irlandais ${ }^{55}$. Cet exemple montre clairement la possibilité de faire usage du DIH.

La pratique témoigne cependant de l'hésitation dont font preuve les organes d'application de la CESDH à occuper une position claire en la matière. Frowein a pointé cette attitude à l'occasion de la première plainte étatique "Cyprus contre Turkey ", dans laquelle la Commission européenne des droits de l'Homme a certes procédé à l'application de la CG III relative aux prisonniers de guerre de 1974, mais a considéré le contrôle relatif aux violations suivant l'article 5 CESDH (droit à la liberté et à la sécurité) comme inutile ${ }^{56}$. Cette hésitation apparaît de manière récurrente ${ }^{57}$.

43 Dans son arrêt «Loizidou contre Turkey ", la CEDH n'a pas appliqué le DIH, malgré l'existence de violations issues d'une occupation militaire : la plaignante chypriote ne 
pouvait plus faire usage de ses biens dans le Nord de Chypre, après l'invasion turque de 1974. En 1989, elle a déposé une plainte fondée sur le fait que l'interdiction constante d'accès à sa propriété constitue une violation du droit à l'usage de ses biens, selon l'article 1 du Protocole Additionnel 1 à la CESDH. Dans une décision CEDH en date du 28 juillet 1989, la plaignante a finalement obtenu gain de cause ${ }^{58}$.

La question centrale de la décision fut de déterminer qui exerce le pouvoir souverain dans la partie Nord de la Chypre. La Turquie a avancé qu'elle n'était pas concernée par cette affaire en tant que défendeur. Ce rôle reviendrait plutôt à la République Turque de Chypre du Nord (RTCN) qui, en tant qu'Etat indépendant, est responsable de ses propres actes. La CEDH a conclu que le terme « juridiction » employé à l'article 1 de la CESDH ne se restreignait pas au seul territoire national. Il s'agirait plutôt de l'exercice du pouvoir étatique suprême qui pouvait avoir des incidences aussi bien à l'intérieur qu'à l'extérieur du territoire étatique. C'est ainsi qu'un Etat peut, à l'aide d'actes militaires, exercer un contrôle effectif sur un territoire se trouvant hors de son propre territoire national. Le fait de savoir si ce contrôle est exercé par l'intermédiaire de ses propres forces armées ou de celles d'une administration locale subordonnée n'a pas d'incidence. En l'espèce, étant donné que la plaignante ne pouvait plus disposer librement de ses biens suite à leur occupation par les troupes turques, selon l'article 1 de la CESDH c'est la juridiction turque qui est responsable de ces évènements.

Toutefois, la CEDH ne s'est pas prononcée sur le fait de savoir si la RTCN constitue un territoire occupé ou non. Ce qui n'a en revanche pas empêché la Cour de procéder au renvoi à la Résolution S/550/1984 du Conseil de sécurité des Nations-Unies, dans laquelle il est explicitement question de «la partie occupée de la République de Chypre ». Cette contradiction a suscité de nombreux commentaires. Dans son opinion dissidente, le juge Pettiti a estimé que l'ensemble des circonstances entourant l'intervention turque à Chypre n'était pas suffisamment clarifié, notamment en ce qui concerne les problèmes d'occupation et d'annexion qui rendent l'application du DIH nécessaire ${ }^{59}$.

Il convient de suivre cette argumentation. Le jugement dans son ensemble souffre du fait qu'on a mis de côté les difficiles questions de statut. C'est la raison pour laquelle la CEDH a simplement estimé que la protection internationale des droits de l'Homme serait une "préoccupation internationale ». Toujours selon la CEDH, il faudrait faire appel à une explication pragmatique (l'effet utile) ${ }^{60}$. Toutefois, on peut se demander si on ne serait pas parvenu au même résultat en passant par le DIH, ce dernier constituant finalement également une "préoccupation internationale ». L'application de la CG IV aurait consolidé le raisonnement de la $\mathrm{CEDH}$, car la partie nord de Chypre est un territoire occupé et Loizidou a été, contrairement à l'article 49 , chassé de ce territoire, sans qu'on ait démontré que la sécurité de la population ou d'impérieuses raisons militaires l'exigeaient. Ceci est également contraire à l'obligation de respecter la propriété de personnes civiles dans un territoire occupé. La raison pour laquelle la Cour n'a pas fait usage de cet argument reste inexpliquée.

Aux termes des $C G$, les parties à un conflit armé sont liées à leurs engagements même en dehors de leur propre territoire. A la lumière de la décision "Loizidou contre Turkey", la juridiction d'un Etat peut aussi s'exercer à l'extérieur du territoire national. Au vu de ces éléments, le jugement «Bankovic contre Belgium » ${ }^{61}$, dans lequel la CEDH n'a pas pu se décider en faveur d'une interprétation large du terme «juridiction», étonne encore davantage. En effet, ce sont les parents de quatre 
citoyens de la République Fédérale de Yougoslavie, tués lors des bombardements de l'OTAN sur la station radio de Belgrade, qui ont porté plainte. Ils estimaient que ces attaques étaient constitutives d'une violation du droit à la vie, à la liberté d'expression et d'opinion, ainsi que du droit à un moyen de recours utile. En outre, ils justifiaient leur plainte par des obligations issues du DIH, qu'ils estimaient devoir s'appliquer, car d'un côté les Etats de l'OTAN sont des Etats signataires des CG et d'un autre côté les dispositions applicables du PA I ont désormais acquis un caractère de droit international coutumier. La Cour a ignoré cette argumentation relative au DIH. Elle s'est au contraire bornée à ne pas accorder une interprétation large au terme «juridiction ». La plainte fut rejetée pour irrecevabilité. En comparaison avec l'arrêt « Loizidou », ce jugement est surprenant.

48 Les opérations militaires sur le territoire kurde de la Turquie ont fait de nombreuses victimes parmi la population civile, ainsi que d'importants dommages matériels. Toute une série de cas similaires ont été portés devant la Cour de Strasbourg. A cette occasion, on a pu constater les limites d'une telle juridiction internationale qui dépend de l'épuisement des voies de recours interétatiques et de la réparation de violations de droits individuels ${ }^{62}$. Ces procédures ne sont par conséquent pas prévues afin de servir utilement de recours contre de graves violations des droits de l'Homme qui se sont produites en masse ${ }^{63}$.

49 Toutefois, les violations en masse des droits de l'Homme comportent une dimension individuelle. Dans l'arrêt «Ergi contre Turkey " ${ }^{64}$, il s'agissait de la mort d'une civile survenue par hasard lors d'opérations militaires. La Commission a déterminé au cours de son enquête que la planification et l'exécution d'une telle opération devrait se dérouler « (...) pas seulement dans le contexte des cibles apparentes d'une opération mais plus particulièrement lorsque l'usage de la force est envisagé dans des quartiers résidentiels, du point de vue de l'absence de morts et de blessures accidentelles infligées aux autres ${ }^{65}$. A la hauteur de ces exigences, la Commission a estimé que la planification des opérations en question n'avait pas été réalisée avec les soins nécessaires afin d'éviter des victimes parmi la population civile et d'empêcher que le conflit ne s'étende.

50 La CEDH a décidé, concernant la responsabilité de l'Etat relative à cet échec, «de prendre toutes les précautions possibles dans le choix des moyens et des méthodes relatives à une opération de sécurité contre un groupe d'opposants, dans la vue d'éviter ou, tout du moins, de minimiser les pertes de civils ${ }^{66}$. La Cour se réfère ainsi aux dispositions du DIH, afin d'analyser le contenu des obligations relatives aux droits de l'Homme. D'un côté, cette manière de procéder est révélatrice de l'application cumulative des deux corps de droit. D'un autre côté, on a la confirmation du constat déjà réalisé par la $\mathrm{CIJ}$, à savoir que le DIH est la lex specialis, qu'il constitue le droit applicable lors des conflits armés, et qu'il a pour but de régler le déroulement des hostilités. En d'autres termes, tout comme la CIJ qui, lors du contrôle de légalité au sujet d'armes atomiques, n'a pas pu se passer d'une vérification de la conformité de ces systèmes d'armes avec le droit des droit de l'Homme et le DIH, la CEDH doit de même se reporter au DIH, lorsqu'elle traite des actions des forces de l'ordre turques sur les territoires kurdes. Dans son arrêt "Ergi », la CEDH se réfère indirectement au DIH lorsqu'elle donne son avis sur ce que constitue une cible légitime, si une attaque légitime est proportionnelle et si le risque prévisible relativement à des victimes civiles est disproportionné par rapport aux avantages militaires ${ }^{67}$. Le seul fait d'avoir énuméré 
ces éléments constitutifs d'un contrôle montre que l'attention portée au DIH peut être primordiale pour la mise en œuvre du droit des droits de l'Homme.

Dans l'arrêt «Gülec contre Turquie » ${ }^{68}$, il s'agissait de coups de feu tirés à partir d'un char d'assaut, lors d'une manifestation, sur des participants au comportement agressif, et qui ont mortellement blessé le fils du plaignant. La Cour a vérifié si l'utilisation de la violence étatique avait été conforme aux dispositions de l'article $2.2 \mathrm{c}$. Elle a avancé comme critère d'appréciation que la violence devait être proportionnelle au moyen utilisé et au but visé. Une telle mise en balance n'a de toute évidence pas eu lieu du côté des forces de l'ordre car elles ont utilisé de l'artillerie lourde. Elles n'étaient pas équipées des moyens nécessaires pour faire face à des manifestants (canons à eaux, boucliers de protection, balles en caoutchouc ou bombes lacrymogènes). Cette action était d'autant plus condamnable que la province de Sirnak se trouve dans une région dans laquelle l'état d'urgence avait été déclaré et dans laquelle on pouvait s'attendre à l'apparition de troubles. Concernant l'affirmation selon laquelle des terroristes se trouvaient parmi les manifestants, les autorités n'ont pu avancer aucune preuve. L'utilisation massive de violences armées qui ont causé la mort de Gülec, n'était, par conséquent, au sens de l'article 2 , ni inévitable, ni nécessaire, de telle sorte que la Turquie fut condamnée pour violation de la CESDH.

52 L'argumentation de la Cour révèle de nombreux parallèles avec le DIH, à commencer par la circonstance où l'état d'urgence avait été décrété dans la région concernée et que des troubles pouvaient survenir à tout instant. Ces deux éléments impliquent à eux seuls que les normes du DIH peuvent trouver application. On trouve également un autre élément révélateur dans le fait qu'on ait reproché aux forces armées l'absence de formation, d'équipement et l'insuffisance de «règles d'engagement ». Enfin, la Cour relève que de nombreuses personnes ont déjà péri dans le sud-est de la Turquie à cause de «l'insécurité » qui règne dans cette zone. Toutefois les « confrontations violentes et armées " récurrents ne constituent pas une cause de désengagement de l'Etat par rapport aux obligations contenues à l'article $2 \mathrm{CESDH}$.

Les exemples traités témoignent des importants recoupements existant entre la pratique des organes strasbourgeois de protection des droits de l'Homme et le DIH. Ce constat vaut notamment pour les droits qui sont énumérés à l'article 3 des CG, et qui ont été caractérisés en tant que droits de l'Homme auxquels on ne peut déroger. Il convient de souligner tout particulièrement le fait qu'en cas de guerre et d'état d'urgence, l'utilisation de la force armée (au sens de l'article 2 al 2 c CESDH) est considérée comme acceptable. Les Etats peuvent avoir recours à la force pour réprimer une émeute ou une insurrection; le recours à la force, rendu absolument nécessaire, peut même mener à la mort. Outre le fait que cet article connaît une "formulation malheureuse $»^{69}$, il subsiste toujours la question de savoir dans quelles proportions et circonstances on peut procéder à l'utilisation de la force. Les exemples cités ci-dessus montrent que les frontières tracées sont très minces et qu'elles ne sont en réalité pas décrites de manière aussi claire par le droit des droits de l'Homme que par le DIH. Les organes strasbourgeois de protection des droits de l'Homme se faciliteraient la tâche si, lors de l'enquête relative à la dimension concrète de l'utilisation de violence, ils se réfèrent aux critères d'appréciation $\mathrm{du} \mathrm{DIH}$, en particulier aux principes de proportionnalité et de mise en balance et les règles standards issues du droit pénal international correspondant. On peut également se figurer des situations de conflit armé lors desquelles les instruments de protection des droits de l'Homme n'envisagent 
aucun critère d'appréciation en ce qui concerne la légitimité du recours à la force. Il faut alors obligatoirement se reporter au DIH. C'est la raison pour laquelle il faut convenir avec Laursen d'accorder au moins un « potentiel pour l'application future du droit humanitaire international » réduit à la $\mathrm{CEDH}^{70}$.

L'arrêt « Engel », dans lequel la CEDH s'est explicitement référée au DIH, montre qu'il n'existe aucun argument juridique théorique valable contre une telle application directe du $\mathrm{DIH}^{71}$. En l'espèce, il s'agissait de traitement inégal entre des rangs militaires de niveau différent dans le cadre de punitions disciplinaires. La référence à l'article 88 CG I fut saluée en doctrine car cette convention serait si bien acceptée ${ }^{72}$. Cette décision fait apparaître de manière évidente que c'est la prise en compte de facteurs politiques qui a, jusqu'à présent, empêché la CEDH d'appliquer le DIH.

La présente analyse démontre qu'il existe une convergence entre la protection des droits de l'Homme et le DIH. Les deux corps de droit peuvent trouver application afin de garantir la protection la plus complète de l'humanité au sens de la clause Marten. Le principal impact pratique de cette convergence se mesure au niveau des possibilités de mise en œuvre du DIH. Le fait que les mécanismes de mise en œuvre sont insuffisants, et que la rédaction de rapports étatiques de plaintes individuelles ne sont pas envisageables pour le DIH, confère une importance pratique grandissante aux procédures de protection des droits de l'Homme, comme en témoignent les premières décision (hésitantes) à l'occasion desquelles le DIH fut appliqué. Ceci constitue également un argument évident en faveur des conséquences pratiques (et utiles) de la convergence du droit des droits de l'Homme et du DIH. La question de savoir dans quelles mesures les victimes du DIH peuvent porter plainte contre des parties belligérantes sur la base du droit à réparation n'a pas encore été résolue. Un groupe de citoyens yougoslaves, dans l'affaire Varvarin, jugée devant le Landgericht de Bonn, l'a tenté en vain. La Cour a rejeté l'argumentation suivant laquelle des individus pouvaient faire une demande en dommages et intérêts contre un Etat pour cause de violation du DIH à l'encontre d'un Etat devant un tribunal national ${ }^{73}$. Ce constat s'écarte en principe des règles relatives aux droits de l'Homme, ce qui rend les mécanismes de protection des droits de l'Homme pour la mise en œuvre du DIH encore plus intéressants. Toutefois, le débat est loin d'être terminé: concernant ce dernier point, le recoupement grandissant entre le DIH et le droit des droits de l'Homme devra également aboutir à de nouvelles évolutions ${ }^{74}$.

\section{NOTES}

1.. Traduction par Lynn Voegele.

2.. RGBI. 1929 II, p. 97 et suivantes.

3.. BGBI. 1973 II, p. 430.

4.. BGBI, 1993 II, p. 1553.

5.. « Legality of the Threat or Use of Nuclear Weapons, Advisory Opinion », ICJ Reports, $1996, \S 26$. 
6.. " transformed into a branch of human rights law and termed, human rights in armed conflicts » : Cerna C.M., « Human Rights in Armed Conflict: Implementation of International Humanitarian Law Norms by regional Intergovernmental Human Rights Bodies ", Kalshoven F., Sandoz Y. (dir.), Implementation of International Humanitarian Law, Geneva 1989, p. 39.

7.. BGBl. 1992 II, p. 121.

8.. BGBl. 1990 II, p. 1551.

9.. Selon l'article 1, tout être humain âgé de moins de 18 ans.

10.. Heintze H.-J., « Die völkerrechtliche Stellung des Kindes im bewaffneten Konflikt Verfestigung eines unbefriedigenden Standards durch die neue UN-

Kinderkonvention ", in Humanitäres Völkerrecht - Informationsschriften 4, 1991, p. 92-98.

11.. UN-Doc. E/CN.4/1989, S. 55/Add.1, p. 6

12. Cet aspect échappe à Matthe Happold lors de l'appréciation de l'impact de ce protocole, « The Optional Protocol to the Convention on the Rights of the Child on the Involvement of Children ", in Armed Conflict, Horst Fischer, Yearbook of International Humanitarian Law 3 (2000), The Hague 2002, p. 242 et suivantes.

13. C'est ainsi que Gasser se prononce contre une « confusion des deux corps du droit international » « advocating a merger of the two bodies of international law » Hans-Peter Gasser, « International Humanitarian Law and Human Rights Law » in Non-International Armed Conflict : Joint Venture or Mutual Exclusion?, in German Yearbook of International Law 45 (2002), p. 162.

14.. La Sous-commission de la Commission des droits de l'Homme parle également d'une convergence dans sa résolution. 1989/26.

15.. Meron T., « Human Rights » in Internal Strife : Their International Protection, Cambridge 1987, p. 28.

16.. Kälin W. (dir.), « Human Rights » in Times of Occupation: The Case of Kuwait, Berne 1994, p. 27.

17.. BGBl. 1973 II, p. 1569.

18.. BGBl. 1990 II, p. 246.

19.. Blatt D., " Recognizing Rape as a Method of Torture », in New York University Review of Law and Social Change 19 (1994), p. 821 et al.

20.. Kälin W., op. cit., p. 27 et suivantes.

21.. " essential tools for the legal protection of civilians in armed conflicts ", UN-Doc. S/ 1999/957, § 36. Traduction de la Rédaction.

22. Meron T., "The Humanization of Humanitarian Law », in AJIL 94, 2000, p. 244.

23.. Fleck D., « Humanitarian Protection Against Non-State Actors », in: Jochen A.

Frowein et. Al. (Hrsg.), Verhandeln für den Frieden, Liber Amicorum Tono Eitel, Berlin 2003, p. 78 et suivantes.

24.. Legality of the Threat or Use of Nuclear Weapons, op. cit., p. 26.

25.. Il s'agit de l'argumentation suivie par la Malaisie, les Iles Salomon et l'Egypte. Voir Greenwood, p. 253. (Voir note suivante)

26.. Greenwood C. J., « Jus bellum and jus in bello in the Nuclear Weapons Advisory Opinion », in Bisson de Chazournes L., Sands P. (dir.), Cambridge 1999, p. 253.

27. Michael J. Matheson, « The Opinions of the International Court of Justice on the Threat or Use of Nuclear Weapons », in AJIL 91, 1997, p. 423.

28. Reimann H. B., « Menschenrechtsstandard in bewaffneten Konflikten ", in Swinarski C. (Hrsg.), Studies and Essays on International Humanitarian Law and Red Cross Principles in Honour of Jean Pictet, Geneva/The Hague, 1984, p. 773. 
29.. Vöneky S., Die Fortgeltung des Umweltvölkerrechts in internationalen bewaffneten Konflikten, Berlin, 2001, p. 286 et suivantes.

30.. Gardam J., « The Contribution of the International Court of Justice to International Humanitarian Law », in Leiden Journal of International Law 14 (2001), p. 353.

31.. Drewicki K., « The Possible Shape of a Reporting System for International Humanitarian Law: Topics to be Addressed », in Bothe M. (dir.), Towards a Better Implementation of International Humanitarian Law, Berlin, 2001, p. 73 et suivantes.

32.. Une telle réflexion, à laquelle on ne prêta malheureusement pas attention, a été menée par Francoise J. Hampson en 1992 déjà. « Using International Human Rights Machinery to Enforce the International Law of Armed Conflict », in Revue de Droit Militaire et de Droit de la Guerre 31, 1992, p. 118 et suivantes.

33.. Gowlland-Debbas V., " The Right to Life and Genocide: The Court and the International Public Policy », in Bisson de Chazournes L., Sands P. (dir.), Cambridge 1999 , p. 324

34.. Ce fut le cas lors de l'éclatement de l'ex-Yougoslavie. Voir Heintze H.-J., « Notstandsfeste Menschenrechte und bewaffneter Konflikt, Anmerkungen zum Sonderbericht der Föderativen Republik Jugoslawien an den Menschenrechtsauschuss ", in Humanitäres Völkerrecht - Informationsschriften 6, 1993, p. 134.

35.. Nowak M.., CCPR-Commentary, Kehl , 1993, p. 81.

36.. "When faced with situations of armed conflicts, both external and internal, which affect State Parties to the Convenant, the Committee will necessarily examine whethter these parties are complying with all their obligations under the Convenant ", UN-Doc. A/57/40, § 29. Traduction de la Rédaction.

37.. BGBl. 1992 II, p. 1246.

38. Par conséquent, il existe de nombreuses décisions concernant des plaintes individuelles, mais qui, la plupart du temps, n'ont été jugées qu'à la fin de l'état d'urgence, sous un nouveau gouvernement. Svensson-McCarthy A.-L., The International Law of Human Rights and States of Exception, The Hague, 1998, p. 392 et suivantes.

39.. UN-Doc. A/57/40, § 31.

40.. Wolfrum R., « The Reporting System under International Human Rights Agreements - From Collection of Information to Compliance Assistence », in Bothe M. (Hrsg.), op. cit., p. 25.

41.. " examination of the enjoyment or deprivation of the rights set forth in the American Declaration of rights and Duties of Man ». Traduction de la Rédaction.

Voir http://www.wcl.American.edu/pub/humanright/digest/Inter-American/app9213.htm. "(...) the Commission must necessarily look to and apply definitional standards and relevant rules of humanitarian law as sources of authoritative guidance in its resolution of this and other kinds of claims alleging violations of the American Convention in combat situations ». Case 11.137. Inter. Am. C.H.R., $\mathrm{n}^{\circ}$ 55/97, § 161 (1997).

42.. Weisbrodt D., Andrus B., « The Right to Life During Armed Conflict : Disabled Peoples' International v. United States », in Harvard International Law Journal 29, 1988, p. 59.

43.. Zegveld L., « The Inter-American Commission on Human Rights and International Humanitarian Law: A comment on the Tablada Case ", in International Review of the Red Cross, 1998, p. 505.

44.. Case 11.137. Inter. Am. C.H.R., $\mathrm{n}^{\circ}$ 55/97, § 161 (1997), op. cit.

45.. Advisory Opinion OC-1/82, 24 septembre1982. 
46.. Inter-Am.Ct.H.R. (Ser.C), $\mathrm{n}^{\circ} 67$ (2000).

47.. « (...) has only given the Court competence to determine whether the acts and norms of States are compatible with the Convention itself, and not with the 1949 Geneva Convention », Ebenda, § 33. Traduction de la redaction.

48. Kleffner J.K., Zegveld L., « Establishing an Individual Complaints Procedure for Violations of International Humanitarian Law », in Fischer H. (dir.), Yearbook of International Humanitarian Law 3 (2000), The Hague, 2002, p. 388.

49.. Il faut cependant se poser la question de savoir s'il n'est pas désormais possible d'obtenir des dommages et intérêts pour violation du DIH en application du droit civil. Voir Graefrath B., « Schadenersatzansprüche wegen Verletzung humanitären Völkerrechts ", in Humanitäres Völkerrecht - Informationsschriften 14, 2001, p. 110 et suivantes.

50.. Inter-Am. Ct.H.R. (Ser.C) n70, 2000.

51.. "Instead of exoneration the State from its obligations to respect and guarantee human rights, this fact oblige it to act in accordance with such obligations »,Ebenda, § 207.

Traduction de la Rédaction.

52.. Wilson R. J., Perlin J., « The Inter-American Human Rights System: Actvities from Late 2000 Through October 2002 », in American University International Law Review 18, 2002, p. 670 et suivantes.

53.. Künzli J., Zwischen Rigidität und Flexibilität : Der Verpflichtungsgrad internationaler Menschenrechte, Berlin, 2001, p. 110.

54.. Harris D. J., O'Boyle M., Warbrick C., Law of the European Convention on Human Rights, London, 1995, p. 489 et suivantes.

55.. Au lieu de cela, on a affirmé l'existence d'une violation de l'article 4 de la CCPR. Voir « Brannigan and McBride v. » UK, ECtHR Series A 258-B, 26 May 1993, §§ 67-73. 56.. Frowein A.J. « The relationship Between Human Rights Regimes and Regimes of Belligerent Occupation », in Israel Yearbook of Human Rights 28 (1999), The Hague 1999, p. 10 et al.

57.. Reidy A. « The approach of the European Commission and Court of Human Rights to international humanitarian law ", in International Review of the Red Cross 324, 1998, p. 519.

58.. Husheer A., « Die völkerrechtliche Verantwortlichkeit der Türkei für Menschenrechtsverletzungen in Nordzypern », in Zeitschrift für Europarechtliche Studien 1(1998) 3, p. 389.

59.. EGMR 1996, p. 2251.

60. Voir l'argumentation convaincante de Husheer, op. cit.

61.. Application $n^{\circ} 52207 / 99$, Eur.Ct.H.R.

62.. Reidy A., Hampson F., Boyle K., « Gross Violations of Human Rights: Invoking the European Convention on Human Rights in the Case of Turkey ", in Netherlands Quarterly of Human Rights 15, 1997, p. 161 et al.

63.. Heintze H.-J., « Entscheidungen des Europäischen Menschenrechtsgerichtshofs als Politikersatz? ", in Hasse J. et al. (Hrsg.), Menschenrechte, Baden-Baden, 2002, p. 442 et al. 64.. Application $n^{\circ} 23818 / 94$, Eur.Ct.H.R.

65.. « (...) not only in the context of the apparent targets of an operation but, particulary where the use of force is envisaged in the vicinity of the civilian population, with regard to the avoidance of incidental loss of life and injury to others ", Ebenda, $\S$ 145. Traduction de la Rédaction. 
66.. « To take all feasible precaution in the choice of means and methods of a security operation mounted against an opposing group with a view to avoiding or, at least, minimising incidental loss of civilian life », jugement $n^{\circ} 66.1997 / 850 / 1057 . E u r . C t . H . R$. Traduction de la Rédaction.

67.. « Ergi contre Turquie » (66/1997/850/1057) jugement du 28 juillet 1998. Toutes ces conditions sont contenues à l'article 57 Abs. 2 a) ii).

68.. « Gülec contre Turquie », (54/1997/838/1044), jugement du 27 juillet 1998.

69.. Voir Frowein J.A., Peukert W., Europäische Menschenrechts-Konvention. EMRK Kommentar - Kehl-Strassburg-Arlington, Engel, 1996. p. 34. Toutefois, cette construction fondée sur le principe de proportionnalité ne change rien au parallèle qu'on peut établir par rapport au DIH.

70.. Laursen A., « Nato, the War over Kosovo, and the ICTY Investigation », in American University International Law Review, 17, 2002, p. 804.

71.. « Engel v. The Netherlands 5370/72 », jugement du 23 novembre 1976.

72.. Merrills J. G., The Development of International Law by the European Court of Human Rights, New York, 1993, p. 225.

73.. Herrmann P., « Recht auf Leben nicht einklagbar? Das Varvarin-Urteil des Landgerichts Bonn vom 10. Dezember 2003 ", in Humanitäres Völkerrecht Informationsschriften 17, 2004, p. 79.

74.. Ceci s'annonçait déjà avec la décision d'appel du OLG Köln (AZ7U/04) du 28 juillet 2005.

\section{RÉSUMÉS}

Les mécanismes de la mise en œuvre du droit international humanitaire sont beaucoup moins développés que ceux du droit de l'Homme. Néanmoins, il existe un certain chevauchement entre ces deux corps du droit. L'article décrit ce chevauchement et analyse les conséquences du point de vue légal concernant leur mise en œuvre. L'application cumulative des droits de l'Homme et $\mathrm{du}$ droit international humanitaire pose inévitablement la question de la relation qu'ils entretiennent. La Cour internationale de justice a répondu à cette question en reconnaissant la primauté du droit international humanitaire vis-à-vis des droits de l'Homme dans les conflits armés, caractérisant ainsi le premier comme lex specialis. L'examination de décisions d'institutions des droits de l'Homme, comme la Commission et la Cour inter-américaine des droits de l'Homme et de la Cour européenne des droits de l'Homme montre une tendance à privilégier le droit international humanitaire. Bien que la pratique de ces institutions des droits de l'Homme soit limitée, elle ouvre des possibilités supplémentaires aux moyens internationaux limités pour faire respecter le droit international humanitaire par les parties aux conflits armés. Ceci démontre la convergence pratique et utile entre les droits de l'Homme et le droit international humanitaire.

The mechanisms for implementing international humanitarian law are much less developed that those applying to human rights. Nonetheless there is a certain overlap between the two bodies of law. The article describes this overlap and analyses the consequences from a legal perspective as far as the implementation is concerned. The cumulative application of human rights and 
international humanitarian law inevitably raises the question about their reciprocal relationship. The International Court of Justice has given its answer by recognizing the primacy of international humanitarian law over human rights law in armed conflicts, thereby characterising the former as lex specialis. The scrutiny of the decisions of the European Court of Human Rights, the Inter-American Commission as well as the Intern-American Court of Human Rights reveals also the tendency to privilege international humanitarian law. Even if the practice of these institutions of human rights is limited, they nevertheless have opened additional possibilities to the limited international means available to force the parties to armed conflicts to respect international humanitarian law. This shows the practical and useful convergence between human rights law and International humanitarian law.

\section{INDEX}

Mots-clés : conflits, droit, droit international, droits de l'Homme, humanitaire

Thèmes : Conventions de Genève

\section{AUTEUR}

\section{HANS-JOACHIM HEINTZE}

Hans-Joachim HEINTZE est chercheur à la Institute for International Law of Peace and Armed Conflict de la Ruhr University of Bochum (Allemagne). Il préside l'ONG « Association for the Study of the World Refugee Problem » et dirige le projet de recherche intitulé « International Law Aspects of Post-Conflict Rebuilding of States ». 